\title{
On the robustness of measurement of reliability stopping criterion in turbo iterative decoding
}

\begin{abstract}
Measurement of reliability (MOR) stopping criterion is able to terminate early in the low and high signal-to-noise ratio (SNR) while maintaining the bit error rate (BER) performance. However, the performance of MOR is only based on one code structure and hence, the robustness of MOR is still unknown in turbo iterative decoding. Thus, this paper will test the robustness of MOR based on the following parameters: frame size, code structure, channel reliability and code rate. Then, we analyse and compare the average iteration number (AIN) and the BER performance of MOR with the benchmark stopping criterion known as Genie to determine the robustness of MOR. From the analysis, MOR has a BER degradation for low code rate. MOR also fails to perform well if the corret channel reliability is not available at the receiver and this results a large degradation in BER performance. However, MOR has close performance to Genie in terms of BER for various frame sizes, code structures and high code rate with the assistance of correct channel reliability. MOR is also able to save AIN at low SNR as compared to Genie and this can reduce delay and complexity of turbo codes.
\end{abstract}

Keyword: Iterative decoding; Reliability output; Stopping criterion; Turbo codes 